Hospital, Birmingham, Alabama (Snead OC, Miles MV. J Pediat $1985 ; \underline{106}: 323)$. A loading dose of $20 \mathrm{mg} / \mathrm{kg}$ was effective but a marked rise in serum glumatic oxaloacetic transaminase activity occurred in 3 of 7 patients treated, requiring cessation of valproate therapy.

\title{
ICTAL VOMITING
}

Paroxysmal vomiting in 9 patients, 3 children $(7,8$, and 11 years of age) and 6 young adults, is reported from the section of Epilepsy and Clinical Neurophysiology, Cleveland Clinic, Cleveland, $\mathrm{OH}$. Amnesia for the episodes occurred in 8 of the 9 patients. Other ictal phenomena prior to the vomiting included staring, automatisms, eye-blinking, grimacing, eye-rolling, chewing, and swallowing. After vomiting, only one patient regained normal alertness immediately. Four patients had temporal lobectomy, the pathology specimens showing mild inflammatory changes, gliosis, and neuronal heterotopia. (Kramer RE et al. Ictus emeticus: an electroclinical analysis.

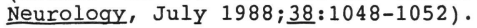

COMMENT. The authors note that reports of ictal vomiting recorded electrographically are scarce and that 13 of 14 cases published have an onset or lateralization of EEG findings to the right hemisphere. In one case the vomiting was induced by photic stimulation. Another case may be added to this list from the French literature (Giroud $M$ et al. Un symptome critique epileptique rare: le vomissement. Arch Fr Pediatr 1987;44: 231-4). In this 9 year old boy, the paroxysmal episode of vomiting was synchronized with an epileptiform discharge in the left fronto-temoral area on the EEG. That cyclic vomiting may represent a form of epilepsy in children was proposed in a report of 33 patients, 7 (218) having a history of grand mal or complex partial seizures in addition, and 25 (76\%) with seizure discharges in the EEG, some focal and predominantly in the temporal lobe. (Millichap JG, Lombroso CT, Lennox WG. Pediatrics 1955;15:705). The EEG was not recorded at the time of the vomiting and, in retrospect, some of our cases may have been more correctly classified as migraine.

\section{SEIZURE RECURRENCE AFTER MEDICATION WITHDRAWAL}

The relapse rate after withdrawal of antiepileptic medication was investigated in 146 children with epilepsy seen at the Dept of Child Neurology, University Hospital, Rotterdam, and Research Unit for Clinical Neurophysiology, Westeinde Hospital, The Hague, The Netherlands. The cumulative probability of remaining seizure-free after a 2 year period of control and normalization of the EEG was 75\%. Three-quarters of relapses occurred during the withdrawal period and in the following 2 years. A significantly higher relapse rate was present in girls and with seizures of known etiology. In patients with partial epilepsy, recurrence may be predicted by the presence of focal neurological signs and/or mental retardation, female sex, a positive family history for epilepsy, and polytherapy. In those with primary generalized epilepsy, no predictive factor was uncovered. The recurrence rate did not change between groups of children who were treated for 2, 3, 4, or 5 years before withdrawal was attempted and EEG epileptiform abnormalities had disappeared. 